\title{
Effect of stimulus duration on stereo and vernier displacement thresholds
}

\author{
J. M. FOLEY and C. WILLIAM TYLER \\ University of California, Santa Barbara, California 93106
}

\begin{abstract}
Stereo and vernier thresholds were determined as a function of stimulus duration in both misalignment and displacement paradigms. All four thresholds increase as stimulus duration decreases. The increase is greatest for the stereo displacement threshold, less for the vernier displacement threshold, and least for the misalignment thresholds. The results are consistent with the hypothesis that depth signals are averaged over time.
\end{abstract}

Tyler and Foley (1974) have distinguished two kinds of stereo and vernier threshold paradigms. One is the displacement paradigm in which a vertical target line is first presented in alignment with the reference (and fixation) line; it is displaced laterally or in depth and then returned to its original position. The displacement has a rectangular temporal waveform. The second paradigm is the misalignment paradigm in which the target line is simply flashed in a misaligned position with respect to the reference line. Tyler and Foley determined stereo and vernier thresholds with both paradigms. They found that, at a duration of $100 \mathrm{msec}$, the stereo displacement threshold was three or more times higher than either the vernier displacement threshold or the stereo and vernier misalignment thresholds. Since the stereo displacement threshold is the sum of the angular displacements in the two eyes, this means that at stereo displacement threshold the displacement in each eye is 1.5 or more times the displacement at vernier threshold. This contrasts with the misalignment paradigms, which yield approximately equal thresholds (Stigmar, 1970; Tyler \& Foley, 1974), which means that in the stereo condition the offset in each eye is about half the vernier offset at threshold. This suggests the generalization that, when a disparate stimulus is presented in close temporal adjacency with a zero-disparity stimulus, the disparity at threshold must be larger than it is without the zero-disparity stimulus.

Various processes might underlie this kind of interaction. One possibility is that the depth-signals produced in the visual system by stimuli that are in the same direction and are closely adjacent in time are averaged in some sense so that the depth signal produced by the two stimuli presented in rapid temporal

This research was supported by NIH Research Grant EY-00666 from the National Eye Institute. We thank Theodore H. Applebaum for assistance in conducting this experiment. C. W. Tyler is now at Smith-Kettlewell Institute of Visual Sciences, 2232 Webster Street, San Francisco, California 94115. sequence has a value intermediate to what it would be for either stimulus alone. It is plausible that the weight of each depth signal is related to the total light energy at the disparity producing that depth signal. If there is such a depth-signal averaging process, then the final depth signal (and threshold values that depend on it) will be sensitive to the temporal parameters of the stimulus sequence. In particular, if disparity is modulated in time, the modulation of the final depth signal will decrease as the frequency of modulation increases. Also, as one stimulus is made shorter within the integration time, the final depth signal will approach the depth signal associated with the other stimulus.

It is important to distinguish between the temporal averaging of depth signals proposed here and the time-intensity tradeoff in visual detection (Bloch's law). As the duration of a light flash increases, the luminance threshold decreases in inverse proportion. Up to a critical duration of $40-100 \mathrm{msec}$, the threshold depends only on the total energy in the stimulus. In such light-detection experiments, stimulus energy is the factor that limits performance. In contrast, stereo misalignment thresholds are independent of luminance as long as contrast is above a minimal level (Ogle \& Weil, 1958). It is not known whether vernier thresholds have this same independence, but, in view of the close relation between performance on the two tasks, it would not be surprising if they did. Stereo and vernier thresholds are limited by the visual angle between the critical contours. These thresholds also decrease as duration increases, but this decrease is far less than proportional to duration, and, at least in the stereo case, it does not depend on stimulus energy. According to our hypothesis, in the displacement paradigm stimulus energy does affect thresholds, not because visibility is the factor limiting performance, but because the weight of the nonzero disparity signal in determining the final depth signal depends on its energy. In detecting lights, energy is integrated. According to the depthsignal averaging hypothesis, in detecting stereo 
offsets, depth signals are averaged with weights related to energy.

Apart from the Tyler and Foley (1974) study, there are a few other experiments in the literature which suggest depth averaging. Richards (1972a) showed that stimuli of different disparities, presented simultaneously in spatially adjacent regions, interact in a way consistent with depth-signal averaging. Kaufman, Bacon, and Barroso (1973) showed that when the two half images of a Julesz pattern were projected to both eyes, so that in effect a zero disparity and a nonzero disparity were simultaneously present, the perceived depth varied continuously as the relative intensities of the half-images in one eye were varied. Foley (1976) showed that, if two vertical lines are presented, one in front and the other an equal disparity behind a fixation point, a single depth will be seen which varies continuously as a function of the luminance ratio of the two lines. In a paradigm more similar to the displacement one, Richards (1972b) showed that the perceived amplitude (in equivalent disparity units) of sinusoidal or squarewave motion in depth is at most about half the stimulus amplitude. In comparison, if disparities of the same magnitude (1 deg) are briefly flashed, effective disparity is approximately equal to physical disparity (Foley, Applebaum, \& Richards, 1975). In the same study, Richards (1972b) found that for frequencies above about $1 \mathrm{~Hz}$ perceived depth amplitude decreases sharply as frequency increases, with no depth being seen for frequencies above $6 \mathrm{~Hz}$. This is consistent with the depth-signal averaging hypothesis and suggests an integration time of about 500 msec. In a more recent study, Beverley and Regan (1974) measured thresholds for seeing motion in depth. Their stimulus was a dot pattern, the central portion of which underwent square-wave displacement with a different frequency in each eye. The threshold was found to be proportional to the higher frequency (which is half the reciprocal of the maximum duration of a disparity) for frequencies within the range of 1.7 and $8 \mathrm{~Hz}$. Tyler (1971) found thresholds for sinusoidal movement in depth to be consistently higher than thresholds for frontal plane motion. As frequency increased above $1 \mathrm{~Hz}$, both thresholds increased, although the sensitivity loss was not as marked as in the two studies just cited.

The high-frequency loss in sensitivity to frontal plane motion found by Tyler might be interpreted in terms of direction (or position) signal averaging. However, Tyler and Foley (1974) found no difference between vernier displacement thresholds and misalignment thresholds at a duration of $100 \mathrm{msec}$. This means that, if there is an averaging process of direction signals, it has different parameters than depth averaging. Recent experiments by Westheimer and Hauske (1975) demonstrate that the presentation of an interfering line or lines up to $150 \mathrm{msec}$ after the vernier lines increases vernier thresholds. However, these effects are not readily interpreted in terms of direction signal averaging, since they are highly dependent on the distance of the interfering stimuli, being maximal at a distance of 3-6 min arc. Spatial-temporal interference could not, however, account for the difference between stereo and vernier thresholds found by Tyler and Foley (1974). Consequently, there must also be a process specific to the depth system, such as the depth averaging process described above. If spatial-temporal interference and depth averaging operate in sequence, then both vernier and stereo displacement thresholds will increase as duration decreases, but the increase in stereo displacement threshold will be greater.

As was noted above, even in misalignment paradigms stereo and vernier thresholds increase as duration decreases. This result has been obtained for stereo thresholds by Ogle and Weil (1958) and Shortess and Krauskopf (1961). In both studies, the threshold was found to change by a factor of about 2 for each log unit change in stimulus duration up to $1 \mathrm{sec}$. A similar change was found by Keesey (1960) for vernier thresholds. One subject common to the experiments showed stereo thresholds higher than vernier thresholds by a factor of about 2 . The increase of these misalignment thresholds as stimulus duration decreases remains unexplained. Generally, when visual performance improves with stimulus duration, the performance is sensitive to the luminance and contrast of the stimulus, and reciprocity holds between luminance and duration for durations below some critical value. This is the case for light-increment detection (Graham \& Kemp, 1938) and acuity measured with the Landolt ring (Kahneman, 1966). In these cases, increasing duration appears to have the same effect as increasing contrast. The stereo threshold, however, is independent of contrast above a minimal level. This has been shown for positive contrast by Ogle and Weil (1958) and for negative contrast by Lit, Finn, and Vicars (1972). Ogle and Weil attributed the improvement to involuntary eye movements that are independent in the two eyes and that increase in average amplitude with exposure time. However, when images are stabilized on the retina, stereo (Shortess \& Krauskopf, 1961) and vernier (Keesey, 1960) thresholds remain unchanged.

The importance of the duration effect on misalignment thresholds for the present study is that the averaging hypothesis requires that the increase in displacement thresholds be greater than the in- 
crease in misalignment thresholds. Therefore, the manner in which all four thresholds vary with target duration is investigated in the present study.

\section{METHOD}

The method was the same as in our previous study (Tyler \& Foley, 1974). The stimuli were vertical lines generated on the face of a fast-phosphor, dual-trace Telequipment oscilloscope laid on its side. These were viewed through orthogonal polarizers so that the line on the left was seen by the right eye and the line on the right by the left eye. The oscilloscope face was at a distance of $2 \mathrm{~m}$ and the two lines were separated by $4 \mathrm{~cm}$ on the screen, thus stimulating a fixation distance of about $125 \mathrm{~cm}$. An upper vertical line segment (reference and fixation line) was continuously present. There were four conditions: stereo displacement, vernier displacement, stereo misalignment, and vernier misalignment. In the displacement conditions, a second vertical line appeared directly below the reference, separated vertically by $6 \mathrm{~min}$ arc. This was displaced either to the left or right for a brief period, then returned to its initial position. In the vernier conditions, the displacement was in the same direction in each eye; in the stereo conditions, it was in the opposite direction. In the misalignment conditions, the lower line was not visible continuously, but appeared briefly in the misaligned position and then disappeared. The upper and lower lines were each $30 \mathrm{~min}$ arc in length and $1.5 \mathrm{~min}$ arc wide. Their luminance was $2 \mathrm{~cd} / \mathrm{m}^{2}$, and they were seen on a dark background.

In all conditions, the subject's head was stabilized by a biting board and he fixated the bottom of the upper vertical line. The momentary offset of a white noise signaled the temporal interval which contained the signal. This noise also had the role of masking auditory cues associated with stimulus change. The subject's task was to respond "left" or "right" in the vernier task and "back" or "front" in the stereo task.

There were five subjects, four males and one female. All were between the ages of 20 and 37 and had acuity of $20 / 20$ and stereo- acuity of $6 \mathrm{sec}$ arc (as measured by Keystone Orthorater tests), with refractive correction in the cases of two of the five.

Thresholds were measured by a forced-choice threshold technique. The initial vernier offset or disparity was twice the estimated threshold. This amplitude was varied in $0.1 \mathrm{log}$ unit steps, being increased after each error and decreased after two successive correct responses. There were 48 triais in a run and the mean amplitude over the last 36 trials was taken as the threshold. This method will yield a threshold stimulus which is responded to correctly on about $70 \%$ of the trials (Rose, Teller, \& Rendleman, 1970). Feedback as to the correctness of each response was given to three of the five subjects.

The design of the experiment was as follows. The displacement thresholds were determined first. There were two tasks, stereo and vernier, and four durations at each. In each session, both stereo and vernier thresholds were obtained at one or two of the four durations. There were four determinations of each of the eight thresholds. For two subjects, J.H. and J.F., the durations were $25,50,100$, and $200 \mathrm{msec}$, and their order was completely randomized. For the three subjects for whom we already had data at $100 \mathrm{msec}$ (Tyler \& Foley, 1974), this duration was not repeated. Thresholds for them were determined at $2,000 \mathrm{msec}$, then later concurrently at 50 and $200 \mathrm{msec}$.

Since our concern here is primarily with the displacement conditions, misalignment thresholds were determined at only two durations, 25 and $200 \mathrm{msec}$ for J.H. and J.F., and 50 and 2,000 msec for L.F. Except for the change in stimulus configuration, the method was the same as in the displacement conditions.

\section{RESULTS}

Geometric mean thresholds are plotted as a function of stimulus duration in Figure 1 for the three subjects who performed in all four conditions. They are plotted on log-log coordinates, as is conventional for this kind of data. The functions are approximately

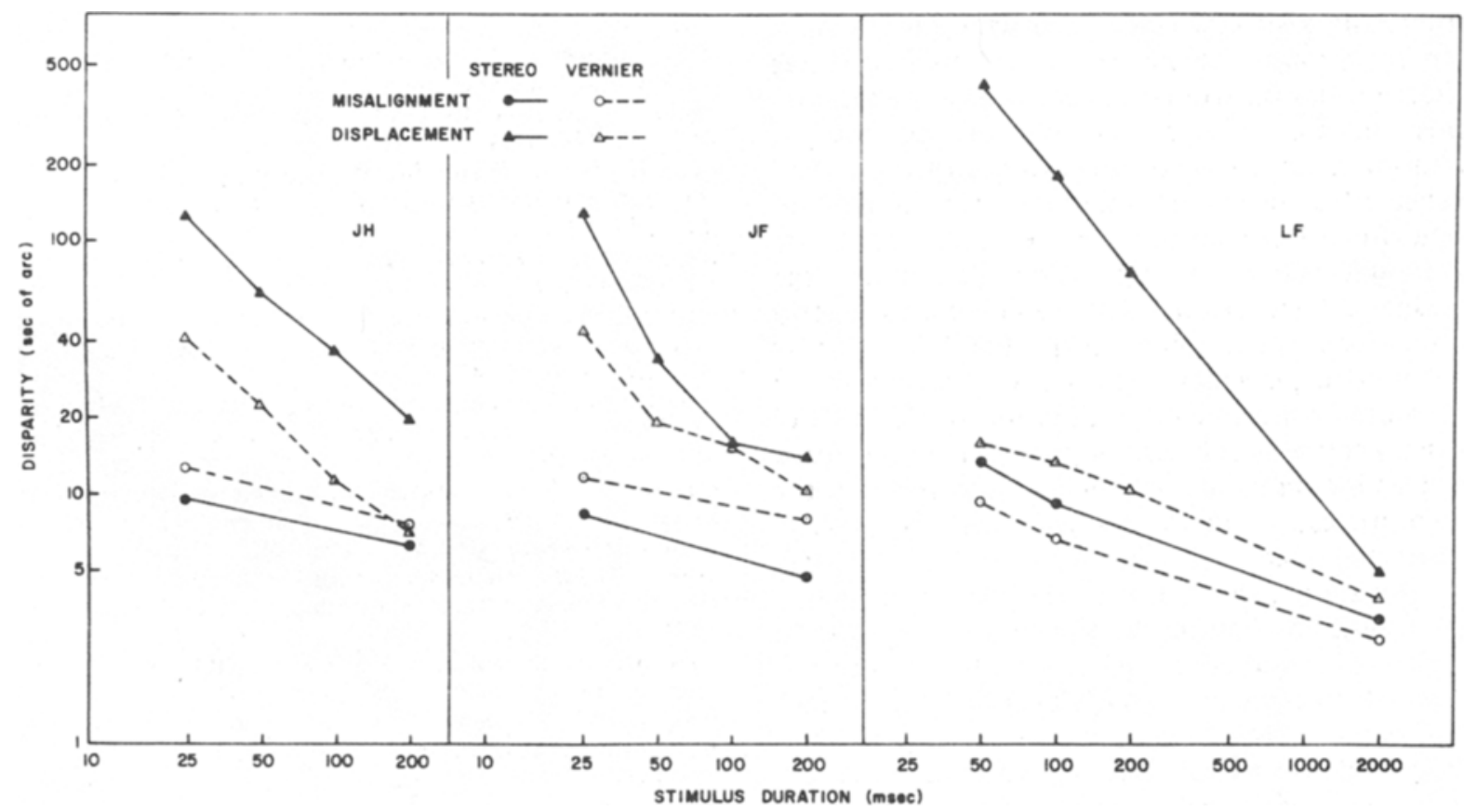

Figure 1. Geometric mean thresholds as a function of stimulus duration for each of the four tasks. 
linear, with the displacement thresholds for J.F. being slightly concave upward. In all cases, thresholds are highest in the stereo displacement condition and lowest in the misalignment conditions. The slopes are in the vicinity of -1 in the stereo displacement condition, -0.5 in the vernier displacement condition (with much more intersubject variability), and -0.2 in both of the misalignment conditions. For J.H., functions for the two displacement thresholds have approximately the same slope and differ by a factor of about 3 throughout the range tested. The results of the two misalignment conditions differ little from each other. For J.H. and J.F.. the stereo threshold is slightly lower; for L.F., the opposite is the case. Standard deviation was roughly proportional to mean threshold in all conditions, being approximately $50 \%$ of the mean in the stereo displacement condition and $30 \%$ of the mean in the other conditions.

\section{DISCUSSION}

Misalignment thresholds obtained here correspond reasonably well with values obtained by Keesey (1961), Ogle and Weil (1958), and Shortess and Krauskopf (1961), although most of their subjects showed a greater increase in threshold as exposure time decreased. One methodological difference which may have contributed to this is that here the reference line was continuously visible, whereas in the studies cited both lines were exposed briefly, although a fixation point or fixation annulus was continuously visible. Keesey used dark targets on a white background, while the others used positive contrast targets on white background, so apparently neither the sign of the contrast nor the level of adaptation is critical to the occurrence of the effect. Another difference which may have been important was the use of feedback in the present study.

Although relatively high variability and large individual differences make it impossible to specify the functions exactly, it is clear that both vernier and stereo displacement thresholds increase in an approximately linear way as duration decreases and that the increase is substantially greater in the stereo case. This is consistent with the hypothesis that there are two processes operating, one of which is specific to the depth system.

The duration dependence of the vernier displacement threshold continues for durations up to $200 \mathrm{msec}$. This value is consistent with the duration over which Westheimer and Hauske (1975) found spatial-temporal interference in a vernier paradigm.

This spatial-temporal interference might be expected to increase stereo displacement thresholds as well. Stereo displacement thresholds, however, start to increase at longer durations and increase at a greater rate as displacement duration decreases.
Stereo displacement thresholds exceed vernier displacement thresholds by a factor of 3 to 20 at the shortest durations. What is responsible for this difference? While the question cannot be answered definitively, the hypothesis described in the introduction that depth-signals are averaged over time is consistent with this result as well as with other results in the literature. For J.H. and J.F., the results suggest averaging over an interval of $500 \mathrm{msec}$, a value that agrees well with the literature. For L.F., the interval appears to be longer. Work in progress is consistent with the temporal averaging interpretation of the stereo results.

\section{REFERENCES}

Beverley, K. I., \& REgAN, D. Temporal integration of disparity information in stereoscopic perception. Experimental Brain Research. 1974, 19. 228-232.

Foley, J. M. Binocular depth mixture. Vision Research, 1976. in press.

Foley, J. M., Applebaum. T. H.. \& Richards, W. Stereopsis with large disparities: Discrimination and perceived depth. Vision Research. 1975, 15, 417-421.

GrahaM, C. H., \& KEMP, E. H. Brightness discrimination as a function of the duration of the increment in intensity. Journal of General Physiology, 1938, 21. 635-750.

KAKNEMAN, D. Time-intensity reciprocity in acuity as a function of luminance and figure-ground contrast. Vision Research. $1960,6,207-215$.

Kaufman. L., Bacon. J., \& Barroso. F. Stereopsis without image segregation. Vision Research, 1973, 13, 137-147.

KEESEY, U. T. Effects of involuntary eye movements on visual acuity. Journal of the Optical Society of America, 1960, 50 , 769-774.

Lit, A.. Finn, J. P., \& Vicars, W. M. Effect of targetbackground luminance contrast on binocular depth discrimination at photopic levels of illumination. Vision Research, 1972, 12. 1241-1251.

Ogle. K. N., \& WeIl. M. P. Stereoscopic vision and the duration of the stimulus. A.M.A. Archives of Opthalmology. 1958. 59. 4-17.

Richards, W. Disparity masking. Vision Research, 1972. 12. $113-1124$. (a)

Richards. W. Response functions for sine- and square-wave modulations of disparity. Journal of the Optical Society of America, 1972, 62, 907-911. (b)

Rose, R. M., Teller, D. Y., \& Rendleman, P. Statistical properties of staircase estimates. Perception \& Psychophysics. 1970. 8. 199-204.

Shortess, G. K., \& Krauskopf, J. Role of involuntary eye movements in stereoscopic acuity. Journal of the Optical Society of America, 1961.51, 555-559.

STIGMAR. G. Observations on vernier and stereo acuity with special reference to their relationship. Acta Ophthalmologica, 1970, 48, 979-998.

TyLER, C. W. Stereoscopic depth movement: Two eyes less sensitive than one. Science, 1971, 174, 958-961.

Tyler, C. W., \& Foley, J. M. Stereo-movement suppression for transient disparity changes, Perception, 1974, 3, 287-296.

Westheimer, G., \& Hauske, G. Temporal and spatial interference with venier acuity. Vision Research, 1975, 15, 1137-1141.

(Received for publication December 21, 1974; revision accepted May 26, 1976.) 